\title{
Molecular Detection and Evaluation of ML- Resistance M. Pneumoniae Associated with Mutation in 23S RNA Gene among Iranian Patients with Respiratory Infections
}

\author{
Iman Pouladi ${ }^{1}$, Reza Mirnejad ${ }^{2}$, Susan Rostampur ${ }^{3}$, \\ Soghra Viesy ${ }^{4}$, Mohammad Niakan*1
}

\begin{abstract}
Background: Mycoplasma pneumoniae is a common cause of community-acquired pneumonia. The global increased resistance of $M$. pneumoniae strains to macrolide (ML) has become a worrisome health problem. The widespread use of these medications has led to increased rate of reported ML-resistant M. pneumoniae (MRMP) throughout the world. This study was aimed to evaluate the resistance of $M$. pneumoniae against erythromycin due to mutations in the $23 S$ rRNA gene of patients with respiratory infections in Iran.

Methods: In this study, 100 samples of throat swab from a patient with respiratory problems were collected. After the cultured of all samples in M. pneumonia-specific PPLO medium, PCR technique was performed with specific primers. Afterwards, the broth micro-dilution MIC assay was employed. Finally, the PCR product of the $23 S$ $r R N A$ gene was sequenced to detect mutations of domain $\mathrm{V}$ in $23 S r R N A$ gene of MRMP.

Results: It was found that 17 cases $(17 \%)$ were positive for mycoplasma genus and six cases $(6 \%)$ positive for $M$. pneumoniae species. Also, analysis of the sequence of $23 S r R N A$ gene, revealed that one of the samples had mutations at positions A2431G and G2491A. All positive samples M. pneumoniae with $23 S$ $r R N A$ gene were sensitive to erythromycin.

Conclusions: These use of these antibiotics should be limited to prevent the emergence of MRMP in Iran.
\end{abstract}

Keywords: Erythromycin, Macrolide resistance, Mycoplasma pneumoniae, 23S rRNA gene.

\section{Introduction}

Mycoplasma pneumoniae (M. pneumoniae) is one of the common causes of upper and lower respiratory tract infections and is a major cause of community-acquired pneumonia (CAP) and atypical pneumonia which is responsible for mortality and morbidity among children and adults (1-4). Since $M$. pneumoniae lacks a cell wall, the infection is primarily treated with macrolide antibiotics (MLs), tetracycline, and fluoroquinolones (5-7). Macrolides like erythromycin, azithromycin and clarithromycin are commonly considered as the first-line treatment of M. pneumoniae infections, because alternative antibiotics, such as tetracycline and fluoroquinolones have side effects $(8,9)$. In addition to antimicrobial activity against $M$. pneumoniae, MLs have anti-inflammatory effects against $M$. pneumoniae infections, and are pharmacologically inhibitors of cytokines, particularly IL-8 (10, 11). Because of limited therapeutic options, the global increase in resistance of $M$. pneumoniae to MLs is a major 
health concern worldwide. Moreover, the widespread application of these drugs has led to an elevated rate of ML-resistant $M$. pneumoniae (MRMP) throughout the world (7, 12, 13). MRMP infection has been shown to cause prolonged treatment, persistent cough, and an elevated time of fever reduction compared with those infections sensitive to treatment $(14,15)$. Currently, the MRMP has been reported more than $90 \%$ in Asia and $26 \%$ in Europe $(7,8,16)$. Undoubtedly, the emergence of MRMP is a serious health threat due to the widespread use of macrolide antibiotics in the treatment of $M$. pneumoniae infections, especially in Asia in recent years. Thus, it has attracted the attention of many scientists (13). The mechanism of resistance of $M$. pneumoniae to ML antibiotics has been a point mutation (nucleotide displacement) of domain $\mathrm{V}$ in certain positions in $23 S$ rRNA gene of MRMP $(17,18)$. In recent years, a number of previous studies, exposed strains of $M$. pneumoniae strains to macrolides, and proposed that all strains with resistance mutations displayed high levels of resistance to ML antibiotics in broth dilution experiments. After Specific Primer-Polymerase Chain Reaction (SSP-PCR) and analysis of the sequences of domain $\mathrm{V}$ of the 23S rRNA gene in some positions, such as A2063G, A2064G, and A2064C, a series of A-to-G or A-to-G nucleotide displacements were identified. Therefore, resistance to MLs in M. pneumoniae can occur due to point mutations in the $23 S$ rRNA gene (10, 17, 19). Therefore, this study was aimed to evaluate the resistance of $M$. pneumoniae against erythromycin due to mutations in the $23 S$ rRNA gene of patients with respiratory infections in Iran.

\section{Materials and methods}

This study was approved by the ethics committee of Shahed University of Medical Sciences, Tehran, Iran.

\section{Sampling}

One hundred samples of throat swab were collected from patients with atypical pneumonia referring to Mostafa Khomeini and KhatamolAnbia hospitals in Tehran province in 2018. All included patients were informed orally of the purpose and experimental procedures of the study and signed the consent form. For sampling, individuals diagnosed with clinical symptoms of respiratory infections by a lung specialist, such as weakness, lethargy, fatigue, persistent headache and dry cough, shortness of breath, sputum production, muscle pain, and no antibiotic use during the past month were targeted for recruitment. Samples were stored in transport pleuropneumonia-like organisms (PPLO) broth medium (Merck, Germany) and moved to the laboratory.

\section{Culturing}

One $\mathrm{ml}$ of the transport medium was transferred following passing through the $0.45 \mu \mathrm{m}$ filter in the main Glucose PPLO broth medium, containing $20 \%$ horse serum, $0.3 \%$ yeast extract, $0.3 \%$ meat extract, $0.33 \mathrm{ml}$ penicillin, $0.5 \mathrm{ml}$ polymyxin $\mathrm{B}$, and $0.5 \mathrm{ml}$ amphotericin $\mathrm{B}$ in $5-10 \% \mathrm{CO} 2$, and were then incubated at $35^{\circ} \mathrm{C}$ for 3 weeks (20).

\section{DNA extraction and PCR}

The M. pneumoniae (ATCC: 29342) used as standard strain and was provided by Molecular Biology Research Center of Baqiyatallah University of Medical Sciences. DNA was extracted using a Kit (Roche, Germany) from the cultures and were subjected to PCR to identify Mycoplasma genus and M. pneumoniae strain with $16 S r R N A$ and $P 1$ primers, respectively (Table 1).

Table 1. Primers sequences used in this study

\begin{tabular}{|c|c|c|c|}
\hline Gene & Sequence & Product Size (bp) & Reference \\
\hline \multirow{2}{*}{$16 S$ rRNA } & F primer: 5' -ACTCCTACGGGAGGCAGCAGT-3' & \multirow{2}{*}{$713 b$} & \multirow{2}{*}{$(21)$} \\
\hline & R primer: 5' TGCACCATCTGTCACTCTGTTAACCTC-3' & & \\
\hline \multirow{2}{*}{$P 1$} & F primer: 5'- AAAGGAAGCTGACTCCGACA-3' & \multirow{2}{*}{ 450bp } & \multirow{2}{*}{ (21) } \\
\hline & R primer: 5'-TGGCCTTGCGCTACTAAGTT-3' & & \\
\hline \multirow{2}{*}{$23 S r R N A$} & Mp-F1: 5'- TAACTATAACGGTCCTAAGG - 3' & \multirow{2}{*}{ 793bp } & \multirow{2}{*}{ (22) } \\
\hline & Mp-R1: 5'-CGCTACAACTGGAGCATAAGA - $3^{\prime}$ & & \\
\hline
\end{tabular}


Table 2. Temperature protocol relates to the cycle of the thermocycler for the P1 gene and the $23 S \mathrm{r} R \mathrm{NA}$ gene.

\begin{tabular}{|c|c|c|c|c|c|}
\hline Gene & Pre Denaturation & Denaturation & Annealing & Extension & Final Extension \\
\hline \multirow{3}{*}{$\begin{array}{c}\text { Genus P1 } \\
\text { M. pneumoniae }\end{array}$} & $94^{\circ} \mathrm{C}$ & $94^{\circ} \mathrm{C}$ & $56^{\circ} \mathrm{C}$ & $72^{\circ} \mathrm{C}$ & $72{ }^{\circ} \mathrm{C}$ \\
\hline & $5 \mathrm{~min}$ & 35 seconds & 40 seconds & 45 seconds & \multirow[t]{2}{*}{$5 \mathrm{~min}$} \\
\hline & & & 35 cycles & $\longrightarrow$ & \\
\hline \multirow{3}{*}{$\begin{array}{c}\text { Genus } 23 S \text { rRNA } \\
\text { M. pneumonia }\end{array}$} & $94^{\circ} \mathrm{C}$ & $94{ }^{\circ} \mathrm{C}$ & $55^{\circ} \mathrm{C}$ & $72^{\circ} \mathrm{C}$ & $72^{\circ} \mathrm{C}$ \\
\hline & $4 \min$ & 45 seconds & 45 seconds & 50 seconds & \multirow[t]{2}{*}{$5 \mathrm{~min}$} \\
\hline & & $\leftarrow$ & 33 cycles & $\rightarrow$ & \\
\hline
\end{tabular}

The PCR reaction with a final volume of $25 \mu 1$ was performed according to the protocol shown in Table 2 for the P1 and 23S rRNA genes in $M$. pneumoniae. Finally, PCR reaction products were electrophoresed. At the end of the PCR step, the samples that were positive for $23 S$ rRNA gene were sequenced to evaluate for the presence or absence of mutations in domain $\mathrm{V}$ of $23 \mathrm{~S} r R N A$ associated with ML resistance, and was analyzed the sequences of $23 S$ rRNA genes.

\section{Minimum inhibitory concentration (MIC)}

The MIC was carried out using micro-dilution of Glucose PPLO broth contains phenol red as a $\mathrm{PH}$ indicator, and $M$. pneumoniae growth change the color of the medium purple to yellow. This medium was containing $25 \%$ horse serum, $0.3 \%$ yeast extract, and $0.3 \%$ meat extract. We used macrolide class antibiotics (erythromycin). Antibiotic susceptibility test was conducted to distinguish between sensitive and clinically resistant strains according to the Clinical and Laboratory Standards Institute (CLSI) criteria (23). The assay was run in in 96well plates. Then, plates were incubated for 5-6 days at $37{ }^{\circ} \mathrm{C}$ and the MIC for erythromycin was determined based on color changes.

\section{Results}

One hundred patients with respiratory infections were recruited in this study, of whom 48 (48\%) were males and $52(52 \%)$ were females. The average age of the participants was 53.62 years, ranging from 17 years to 85 years. According to the findings of our study, 14 subjects (14\%) had M. pneumoniae colonies grown on PPLO agar. The length of 16S rRNA gene fragment of Mycoplasma strain is about 713 bp. Of 100 examined samples obtained from electrophoresis, this fragment was observed in the standard strain in 17 samples (17\%). Also, in positive specimens in PCR, the 450 bp DNA fragment of P1 gene was found in our standard strain among 6 subjects using P1-specific primer of M. pneumoniae. According to the results of the PCR test, 6 cases (6\%) were infected with M. pneumoniae. Figure 1 (B), illustrates the electrophoretic evaluation of $23 \mathrm{~S}$ rRNA in samples positive for M. pneumoniae infection. Using the 23S rRNA-specific primer, the 793 bp fragment was observed in the standard (ATCC: 29342) strain in 6 samples.

Analysis of the 23S rRNA gene sequence, revealed that only one of the samples had point mutation at A2431G and G2491A positions (Fig. 2).

Measuring the MIC in all positive samples for M. pneumoniae using broth micro-dilution method, showed that all specimens were sensitive to erythromycin. Moreover, no ML resistance was reported (Table 3). Therefore, mutations in domain $\mathrm{V}$ of $23 \mathrm{~S}$ rRNA gene at A2431G and G2491A were not associated with resistance of $M$. pneumoniae against erythromycin. 


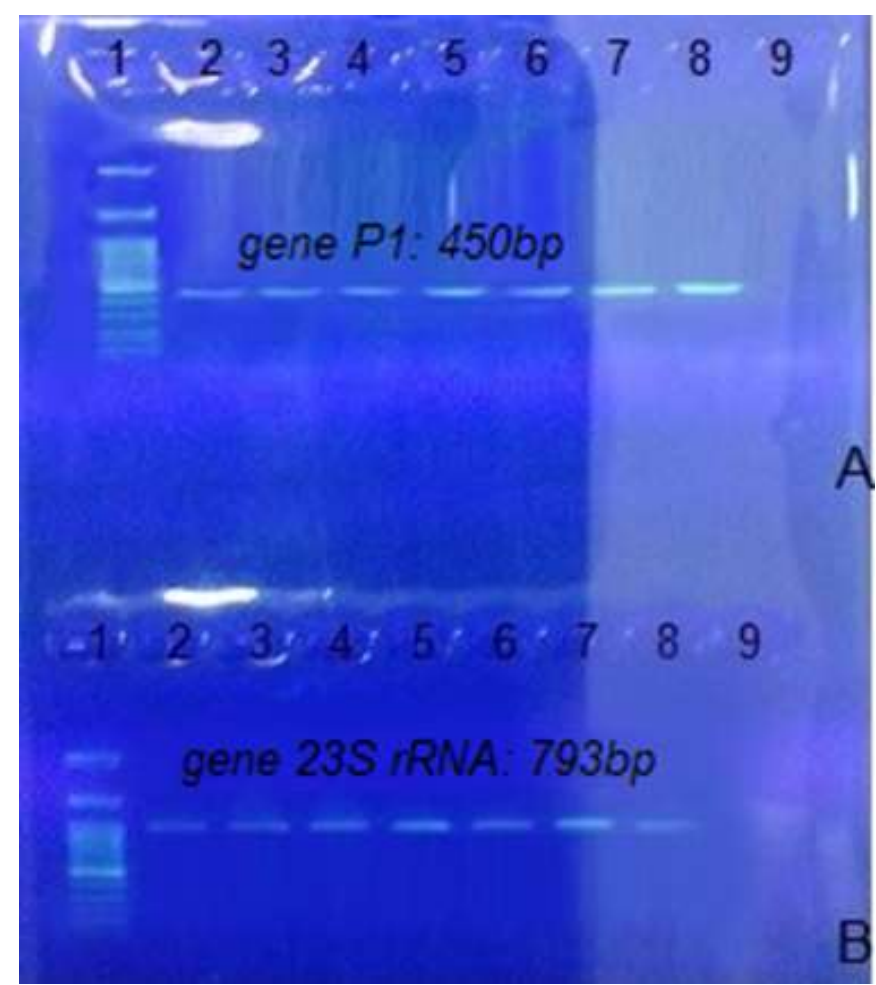

Fig. 1. A) PCR amplification of the $P 1$ gene. Lane 1: Ladder (100 bp), lane 9: negative control, lane 2: positive control (450bp); lane 3-8: positive results. B) PCR amplification of the 23S rRNA gene. Lane 1: Ladder (100 bp), lane 9: negative control, lane 2: positive control (793bp); lane 3-6, 7, and 8: positive results.

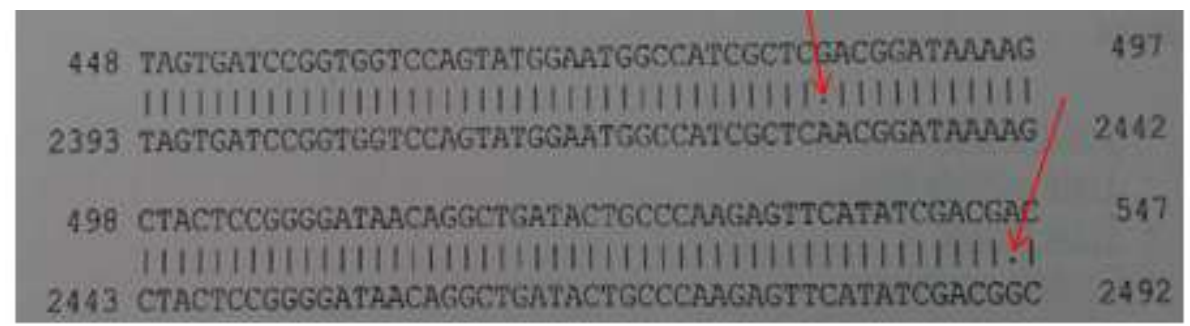

Fig. 2. Schematic representation of a mutation in $23 S r R N A$ genes in online clustalW2 software. The image of both mutations is shown in positions 2431 and 2491.

Table 3. MIC ranges $(\mu \mathrm{g} / \mathrm{ml})$ for Erythromycin antimicrobial against M. pneumoniae.

\begin{tabular}{lll}
\hline Clinical isolate & MIC & Mutation \\
\hline A & 0.0039 & NO \\
\hline B & 0.0039 & Yes \\
\hline C & 0.0156 & NO \\
\hline E & 0.0078 & NO \\
\hline F & 0.0039 & NO \\
\hline Reference strain $\boldsymbol{A T C C}: \mathbf{2 9 3 4 2}$ & 0.0156 & NO \\
\hline MIC range for all isolates & 0.0039 & NO \\
\hline
\end{tabular}




\section{Discussion}

Macrolides are recommended for the treatment of $M$. pneumoniae which are usually considered as the first selective agent for the treatment of $M$. pneumoniae infection (24). With widespread use of these drugs, ML resistant isolates have been reported worldwide $(24,25)$. In 1970 , the first clinical case of erythromycin-resistant $M$. pneumoniae was reported in a girl (13). ML resistance is associated with a point mutation in domain $\mathrm{V}$ of $23 S$ rRNA gene and ribosomal proteins L22 and L14. Mutation affecting $23 S$ $r R N A$ gene at $\mathrm{A} 2063 \mathrm{G}$ position is the most common mutation, followed by $\mathrm{A} 2064 \mathrm{G}$ position, which is mostly responsible for significant ML resistance in $M$. pneumonia $(13,26)$. The present study did not show the presence of MRMP in patients with respiratory diseases caused by $M$. pneumoniae in Tehran and also A2063G mutation, which is the most common cause of high macrolide resistance in patients with $M$. pneumoniae pneumonia. In this study MRMP in patients with respiratory diseases $(0 \%)$ were reported. This is very low compared to recent cases in children in Japan $(87.1 \%)$ and China (97.0\%) (27). The study groups are different thus limiting the comparison. High levels of ML drug resistance in $M$. pneumoniae in East Asia are associated with widespread use of macrolides (28). A study by Waites et al in the United States found that M. pneumoniae ML resistant (MRMP) was associated with clinical symptoms. Between 2015 and 2018, hospitals in eight states collected and stored respiratory samples which were positive for the presence of $M$. pneumoniae PCR test was performed to detect mutations in the 23SrRNA gene of these samples These mutations were associated with macrolide resistance During this study, MRMP was reported in 27 strains of 360 samples (7.5\%). The prevalence of MRMP was significantly higher in the south and east $(18.3 \%)$ than in the west (2.1\%). And A2063G were identified as the dominant mutation in the $23 S$ rRNA gene (29). In the study, Zeng Zhang et al., Out of 55 positive samples, 25 (45\%) were hospitalized. Most of these patients were male $(58 \%)$ aged $7-8$ years. $72 \% \quad(25 / 18)$ of hospitalized patients had radiographic findings consistent with pneumonia and some cases were hospitalized for up to 4 weeks. The results of pathogen diagnosis showed that $M$. pneumoniae (P1 gene) was the causative agent of the disease in this outbreak and all strains had an $A$ to $G$ point mutation at position 2063 (30). In the study of Dongxing Guo et al., Out of 178 collected samples, 164 (90.85\%) cases of M. pneumoniae positive were reported, and in 149 of 164 positive cases of ML-resistant M. pneumoniae (MRMP) using allele-specific real- time PCR was detected, while 153 cases of $M$. pneumoniae were reported positively using nested PCR method, in 110 cases (110/153) of these samples MRMP was detected (31). In a study by Brown et al. (2015) in England ML related mutations were reported in positive samples of $M$. pneumoniae found that 43 samples of the total 60 positive specimens for $M$. pneumoniae had mutations in domain $\mathrm{V}$ of $23 \mathrm{~S}$ rRNA gene, and ML resistance was found in four samples (9.3\%) (26). The results of this study are not consistent with the present study. Results of the analysis of the sequence of $23 S$ rRNA gene showed that point mutations at $\mathrm{A} 2431 \mathrm{G}$ and G2491A positions were reported only in one specimen. In addition, the broth microdilution MIC method revealed that all samples positive for $M$. pneumoniae, were sensitive to erythromycin, and no ML resistance was reported in specimens. The rate of MRMP among study participants was $(0 \%, 6 / 0)$. The prevalence of MRMP was reported as $(2 \%, 50 / 1)$ in Switzerland, $(87.1 \%, 202 / 176)$ in Japan, (97\%, 33/32) in China, $(1.2 \%, 167 / 2)$ in Germany, and $(9.8 \%, 51 / 5)$ in France. As a result, in order to control the emergence of ML resistance among $M$. pneumoniae strains, the use of these antibiotics should be restricted in Iran and even should be avoided widespread use.

\section{Acknowledgment}

The authors would like to thank all staff members of the Department of Microbiology of Shahed University of Medical sciences. The authors declare that there are no competing interests. The results described in this paper were part of a thesis. This study was supported by Department of Medical Microbiology, Faculty of Medicine, Shahed University, Tehran, Iran. 


\section{References}

1. Eshaghi A, Memari N, Tang P, Olsha R, Farrell DJ, Low DE, et al. Macrolide-resistant Mycoplasma pneumoniae in humans, Ontario, Canada, 2010-2011. Emerg Infect Dis. 2013;19(9):1525-1527.

2. Babaei Z, Pouladi I, Ashtari A, Azimi G, Niakan M. The Prevalence of Atypical Pneumonia Caused by Mycoplasma pneumoniae (P1 gene) in Patients with Respiratory Infections by Culture and Molecular PCR Methods in Tehran, Iran. Jundishapur Journal of Microbiology. 2019;12(11):e84174.

3. Gdalevich M, Haas EJ, Dukhan L, Katz M, Zelenski V, Moran-Gilad J. Control of a Mycoplasma pneumoniae Outbreak in an Institutional Setting Using Azithromycin Prophylaxis. Front Public Health. 2017;5:366.

4. Xiao L, Ptacek T, Osborne J, Crabb D, Simmons W, Lefkowitz E, et al. Comparative genome analysis of Mycoplasma pneumoniae. BMC Genomics. 2015;16(1):610.

5. Zhang Y, Mei S, Zhou Y, Yang D, Pan T, Chen $\mathrm{Z}$, et al. TIPE2 negatively regulates mycoplasma pneumonia-triggered immune response via MAPK signaling pathway. Nature.2017; 7(1): 13319.

6. Wang L, Feng Z, Zhao M, Yang S, Yan X, Guo $\mathrm{W}$, et al. A comparison study between GeXPbased multiplex-PCR and serology assay for Mycoplasma pneumoniae detection in children with community acquired pneumonia. BMC Infect Dis. 2017;17(1):518.

7. Xu W, Guo L, Dong X, Li X, Zhou P, Ni Q, et al. Detection of Viruses and Mycoplasma pneumoniae in Hospitalized Patients with Severe Acute Respiratory Infection in Northern China, 2015-2016. Jpn J Infect Dis. 2018;71(2):134-139.

8. Kawai Y, Miyashita N, Kubo M, Akaike H, Kato A, Nishizawa $\mathrm{Y}$, et al. Nationwide Surveillance of Macrolide-Resistant Mycoplasma pneumoniae Infection in Pediatric Patients. Antimicrobial Agents and Chemotherapy.2013;57(8):4046-4049.

9. Nakatani M, Shingo Mizunaga S, Takahata M, Nomura N. Inhibitory activity of garenoxacin against DNA gyrase of Mycoplasma pneumonia. J Antimicrob Chemother 2012;67(8):1850-2.
10. Meyer Sauteura P, Bleischa B, Voitb A, Maurerb F, Rellya C, Berger C, et al. Survey of macrolide-resistant Mycoplasma pneumoniae in children with community-acquired pneumonia in Switzerland. Swiss Med Wkly. 2014;144:w14041.

11. Spuesens E, Meijer A, Bierschenk D, Hoogenboezem T, Donker G, Hartwig N, et al. Macrolide Resistance Determination and Molecular Typing of Mycoplasma pneumoniae in Respiratory Specimens Collected between 1997 and 2008 in The Netherlands. J Clin Microbiol. 2012;50(6):1999-2004.

12. Yin YD, Wang R, Zhuo C, Wang H, Wang MG, Xie CM, et al. Macrolide-resistant Mycoplasma pneumoniae prevalence and clinical aspects in adult patients with community-acquired pneumonia in China: a prospective multicenter surveillance study. J Thorac Dis. 2017; 9(10):37743781.

13. Hong JH, Chun JK, Uh Y, Oh KJ, Kim J, Yoon KJ. Two Cases of Mycoplasma pneumoniae Pneumonia with A2063G Mutation in the 23S rRNA Gene in Siblings. Ann Lab Med. 2013;33(1):65-68.

14. Lee KE, Kim KW, Hong JY, Kim KE and Sohn MH. Modulation of IL 8 boosted by Mycoplasma pneumoniae lysate in human airway epithelial cells. J Clin Immunol. 2013;33(6):11171125.

15. Meyer Sauteur P, Unger W, Nadal D, Berger C, Vink C, Rossum A. Infection with and Carriage of Mycoplasma pneumoniae in Children. Front Microbiol. 2016;7:329.

16. Zhao F, Liu G, Wu J, Cao B, Tao X, He L, et al. Surveillance of Macrolide-Resistant Mycoplasma pneumoniae in Beijing, China, from 2008 to 2012. Antimicrobial Agents and Chemotherapy. 2013;57(3):1521-1523.

17. Izumikawa $K$, Izumikawa $K$, Takazono $T$, Kosai K, Morinaga Y, Nakamura S, et al. Clinical features, risk factors and treatment of fulminant Mycoplasma pneumoniae pneumonia: a review of the Japanese literature. J Infect Chemother. 2014;20(3):181-5.

18. Saraya T. Mycoplasma pneumoniae infection: Basics. J Gen FAM Med, 2017;18(3):118-125. 
19. Youn YS, Lee SC, Rhim JW, Shin MS, Kang JH, Lee KY. Early Additional ImmuneModulators for Mycoplasma pneumoniae Pneumonia in Children: An Observation Study. Infect Chemother 2014;46(4):239-247.

20. Guo DX, Hu WJ, Wei R, Wang H, Xu BP, Zhou W, et al. Epidemiology and mechanism of drug resistance of Mycoplasma pneumoniae in Beijing, China: A multicenter study. Bosn J Basic Med Sci. 2019;19(3):288-296.

21. Golmohammadi R, Ataee R A, Alishiri G H, Mirnejad R, Najafi A, Tate M, et al. Molecular Diagnosis of Mycoplasma pneumoniae in Synovial Fluid of Rheumatoid Arthritis Patients. Iranian Journal of Medical Microbiology. 2014; 8(1):1-8. 22. Liu X, Jiang Y, Chen X, Li J, Shi D, Xin D. Drug Resistance Mechanisms of Mycoplasma pneumoniae to Macrolide Antibiotics. Biomed Res Int. 2014;2014:320801.

23. Wayne PA. Clinical and Laboratory Standards Institute: Performance Standards for Antimicrobial Susceptibility Testing: 30th Informational Supplement. United Status: CLSI Document M100-S30; 2020.

24. Morozumi M, Takahashi T, Ubukata K. Macrolide-resistant Mycoplasma pneumoniae: characteristics of isolates and clinical aspects of community-acquired pneumonia. J Infect Chemother. 2010;16(2):78-86.

25. Beikmohammadi H, Pouladi I, Zolfaghari MR, Niakan M. The Prevalence of 23S rRNA
Mutations in ML-Resistant M. pneumoniae Isolates to Clarithromycin in Patients with Respiratory Infections. Reports of Biochemistry \& Molecular Biology. 2020;9(2):157-162.

26. Brown RJ, Macfarlane-Smith L, Phillips S, Chalker VJ, et al. Detection of macrolide resistant Mycoplasma pneumoniae in England, September 2014 to September 2015. EuroSurveil. 2015;20(48):30078.

27.Zhao F, Liu G, Wu J, Cao B, Tao X, He L, et al. Surveillance of macrolide-resistant Mycoplasma pneumoniae in Beijing, China, from 2008 to 2012. Antimicrob Agents Chemother. 2013; 57(3):1521-3.

28. Bebear C, Pereyre S, Peuchant O. Mycoplasma pneumoniae: susceptibility and resistance to antibiotics. Future Microbiol. 2011;6(4):423-31.

29. Waites KB, Ratliff A, Crabb DM, Xiao L, Qin $\mathrm{X}$, Selvarangan $\mathrm{R}$, et al. Macrolide-resistant Mycoplasma pneumoniae in the United States as determined from a national surveillance program. $\mathbf{J}$ Clin Microbiol. 2019;57(11):e00968-19.

30. Zhang, W. Z, Zhang, S. J, Wang, Q. Y, Li, Y. D, Jing, H. B, Hu, G. Y, et al. Outbreak of macrolide-resistant Mycoplasma pneumoniae in a primary school in Beijing, China in 2018. BMC Infectious Diseases. 2019;19(1):871.

31. Guo D, Hu W, Xu B, Li J, Li D, Li S, et al. Allele-specific real-time PCR testing for minor macrolide-resistant Mycoplasma pneumoniae. BMC Infect Dis. 2019;19:616. 